\title{
ARTICLE \\ Brain function during stages of working memory in schizophrenia and psychotic bipolar disorder
}

This article has been corrected since Advance Online Publication and a correction is also printed in this issue.

Anna S. Huang ${ }^{1}$, Baxter P. Rogers ${ }^{2}$, Alan Anticevic ${ }^{3}$, Jennifer Urbano Blackford ${ }^{1,4}$, Stephan Heckers ${ }^{1}$ and Neil D. Woodward $^{1}$

Working memory (WM) is impaired in psychotic disorders and linked to functional outcome. Most neurobiological models emphasize prefrontal cortex (PFC) dysfunction in the etiology of WM impairment. However, WM is composed of multiple processes, including encoding and maintenance, and the delineation of the neurobiology of these sub-processes has not been well characterized in schizophrenia and psychotic bipolar disorder. Functional MRI was obtained during an event-related spatial delayed match-to-sample task from 58 healthy individuals, 72 individuals with schizophrenia and 41 people with bipolar I disorder with psychotic features in order to: 1) characterize neural responses during encoding, maintenance and retrieval stages of WM using complementary region-of-interest and whole brain approaches; 2) determine whether schizophrenia and psychotic bipolar disorder exhibit similar abnormalities in WM-related brain function; and 3) elucidate the associations between WM-related brain function, task performance, and neuropsychological functioning. Both schizophrenia and psychotic bipolar disorder groups showed encoding- and maintenance-related impairments in the posterior parietal cortex (PPC) and frontal eye fields (FEF). BOLD response in the PPC and FEF, during encoding and maintenance respectively, was associated with task performance independent of group. Additionally, encoding-related activation in the PPC correlated with general neuropsychological functioning independent of group. Only encoding-related activation in the right ventral striatum differed between schizophrenia and psychotic bipolar disorder; individuals with schizophrenia showed significantly lower activation than both psychotic bipolar disorder and healthy groups. Our results are consistent with emerging evidence implicating PPC dysfunction in WM impairment and suggest interventions targeting neural activation in PPC may improve WM and neuropsychological functioning across psychotic disorders.

Neuropsychopharmacology (2019) 44:2136-2142; https://doi.org/10.1038/s41386-019-0434-4

\section{INTRODUCTION}

Working memory (WM) is impaired in psychotic disorders, including schizophrenia and bipolar disorder with psychotic features [1, 2]. Associations between impaired WM, functional outcome [3], and genetic vulnerability for psychosis [4-8] underscore the importance of elucidating the neural basis of impaired WM. Informed in large part by non-human primate studies [9-11], most theories posit that WM impairment in psychosis is a consequence of dysfunction within the prefrontal cortex (PFC), dorsolateral PFC specifically [12-15]. These models are broadly supported by neuroimaging studies which often find altered PFC function during WM tasks in schizophrenia $[16,17]$.

Despite the significant attention WM has received in psychosis, especially schizophrenia, several critical knowledge gaps remain. First, it is unclear which component(s) of WM are impaired. WM is often conceptualized as involving at least three stages: encoding, maintenance, and retrieval [11, 18, 19]. Most neuroimaging investigations used tasks such as the n-back, which inherently mix these stages of memory, in addition to placing significant demands on executive functions $[16,17]$. The Sternberg task, which can separate WM stages, has also been used. However, studies in schizophrenia have frequently used a modified version of the Sternberg task which consists of a shorter delay coupled with prolonged retrieval, requiring multiple comparisons with memorized stimuli. While this increases power to detect group differences, as with n-back tasks, it confounds maintenance with retrieval processes $[20,21]$.

A small number of studies have used tasks capable of examining function during specific WM stages, such as the delayed match-tosample (DMTS) paradigm; a task modeled after paradigms used in preclinical animal studies with a well-established neurophysiological basis $[10,11]$. Results from these studies have been mixed, with some identifying encoding-related deficits, and others finding maintenance-related deficits $[19,22-25]$. The inconsistent findings are likely due to several factors, including small sample sizes (most studies included fewer than 20 patients) and inconsistent inclusion of a sensorimotor control condition to isolate WM-related brain regions. Moreover, while the focus is usually on PFC dysfunction, studies that conducted whole brain analyses also found abnormalities in other frontoparietal network regions, namely the posterior parietal cortex (PPC) $[23,26-30]$. Research in healthy individuals suggest that PFC and PPC have different roles in supporting WM, with PFC regions underlying executive functions and PPC supporting maintenance and attention processes (see ref. [31] for

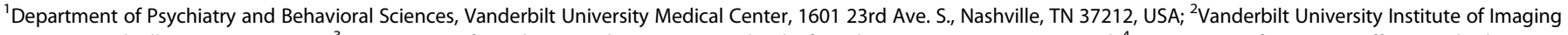

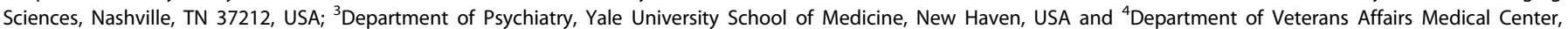
Research Health Scientist, Research and Development, Nashville, TN, USA

Correspondence: Anna S. Huang (anna.s.huang@vumc.org)

Received: 17 April 2019 Revised: 17 May 2019 Accepted: 29 May 2019

Published online: 11 June 2019 
Table 1. Demographics of study participants

\begin{tabular}{|c|c|c|c|c|c|c|c|c|c|}
\hline \multirow[b]{2}{*}{ Variable } & \multicolumn{2}{|c|}{$\begin{array}{l}\text { Healthy Subjects } \\
(N=58)\end{array}$} & \multicolumn{2}{|c|}{$\begin{array}{l}\text { Schizophrenia } \\
(N=72)\end{array}$} & \multicolumn{2}{|c|}{$\begin{array}{l}\text { Bipolar Disorder } \\
(N=41)\end{array}$} & \multirow[b]{2}{*}{$d f$} & \multirow[b]{2}{*}{$X^{2} / t / F$} & \multirow[b]{2}{*}{$\mathrm{p}$} \\
\hline & Mean/n & SD & Mean/n & SD & Mean/n & SD & & & \\
\hline Ethnicity (W:AA:O) & $41: 13: 4$ & & $56: 15: 1$ & & $35: 4: 2$ & & 4 & 5.49 & .240 \\
\hline Age & 29.2 & 9.5 & 27.9 & 9.4 & 31.3 & 12.0 & 2,168 & 1.48 & .232 \\
\hline Paternal Education & 15.1 & 2.9 & 14.8 & 3.9 & 15.3 & 3.1 & 2,162 & 0.10 & .904 \\
\hline SCIP Z score & 0.30 & 0.60 & -0.74 & 0.77 & -0.53 & 0.94 & 2,167 & 30.98 & $<.001$ \\
\hline WTAR & 114.0 & 8.9 & 104.2 & 13.0 & 107.9 & 12.1 & 2,166 & 11.40 & $<.001$ \\
\hline Duration of Illness (years) & - & - & 7.1 & 8.3 & 6.9 & 8.3 & 109 & 0.13 & .901 \\
\hline PANSS Positive & - & - & 15.1 & 8.8 & 12.2 & 9.2 & 111 & 1.61 & .109 \\
\hline APD Dose (mg, in CPZ equivalents) & - & - & 373.0 & 234.4 & 248.1 & 176.3 & 83 & 2.46 & .016 \\
\hline
\end{tabular}

review). Few studies have focused on PPC effects in schizophrenia. A recent study explored PPC deficits associated with WM storage in schizophrenia and found that individuals with schizophrenia were less able to flexibly modulate their PPC activation depending on WM storage requirements, and that the degree of PPC impairment was associated with general cognition [32]. Broadly speaking though, an a-priori interest in PFC dysfunction, coupled with modest sample sizes and study designs that confound different WM processes has biased the literature towards a PFC-centric view of WM impairment in psychosis.

Another important unresolved question is whether schizophrenia and psychotic bipolar disorder show similar neurobiological changes underlying WM impairments. A recent study found increased frontoparietal network activation in both schizophrenia and bipolar disorder, with schizophrenia showing greater activation than bipolar disorder [33]. While this study had a large sample size, they used a n-back task and network analyses, so were not able to investigate separable WM processes, or frontal and parietal regions separately. Another study found the opposite gradient in the dorsolateral PFC, whereby individuals with schizophrenia showed the lowest activation, followed by individuals with bipolar disorder then healthy individuals [34]. This study did not examine regions that differ between all three groups across the whole brain. To our knowledge, no study has so far investigated neural responses to specific WM stages in both schizophrenia and psychotic bipolar disorder.

The current investigation utilized an event-related spatial WM task in a relatively large sample of individuals with psychosis and healthy individuals in order to: 1) characterize neural responses during WM encoding, maintenance, and retrieval; 2) determine if individuals with schizophrenia and psychotic bipolar disorder demonstrate similar abnormalities in WM-related brain activity; and 3) elucidate the associations between WM-related brain activation, task performance, and general cognitive function.

\section{METHODS}

Participants

Sixty-two healthy individuals, 90 individuals with schizophrenia spectrum disorders, and 49 individuals with bipolar I disorder with psychotic features were initially screened for inclusion in this investigation. Twenty-nine subjects were subsequently excluded after being found ineligible for the study or withdrawing consent (healthy: $n=1$; schizophrenia: $n=1$; bipolar disorder: $n=1$ ), and for having a WM accuracy lower than $60 \%$ (healthy: $n=3$; schizophrenia: $n=17$; bipolar disorder: $n=7$ ). Thus, the final sample consisted of 58 healthy individuals, 72 individuals with schizophrenia spectrum disorders (12 schizoaffective disorder, 17 schizophreniform disorder and 43 schizophrenia patients) and 41 individuals with psychotic bipolar disorder (see Table 1 for demographics). Individuals with schizophrenia and psychotic bipolar disorder were recruited from inpatient and outpatient services at the Vanderbilt Psychiatric Hospital in Nashville, Tennessee. Healthy individuals were recruited from Nashville and the surrounding area through advertisement. This study was approved by the Vanderbilt University Institutional Review Board and all participants provided written informed consent prior to participating in this study and received financial compensation.

The Structured Clinical Interview for Diagnosing DSM-IV Disorders (SCID; [35]) was administered to confirm diagnoses in patients and rule out current or past psychiatric illnesses in healthy participants. Clinical symptoms of psychosis and mania were quantified in patients with the Positive and Negative Syndrome Scale (PANSS; [36]), and Young Mania Rating Scale (YMRS; [37]). All study participants were administered the Wechsler Test of Adult Reading (WTAR: [38]), a single wordreading test of estimated premorbid intellectual functioning, and the Screen for Cognitive Impairment in Psychiatry (SCIP; [39]), a brief test of neuropsychological functioning that assesses verbal learning, WM, processing speed, and verbal fluency. Exclusion criteria included estimated premorbid IQ of less than 75 , age less than 18 or greater than 55 , presence of medical illness that would affect study participation (e.g. diabetes, cardiovascular or central nervous system disorder, history of significant head trauma), reported pregnancy or lactation, history of substance abuse/ dependence (3-months in patients; lifetime in healthy individuals), psychotropic drug use (healthy individuals), and any MRI contraindications (e.g. metal implants, claustrophobia).

Spatial Working Memory Task

Participants performed a spatial WM task virtually identical to that used in several prior neuroimaging investigations of spatial WM 


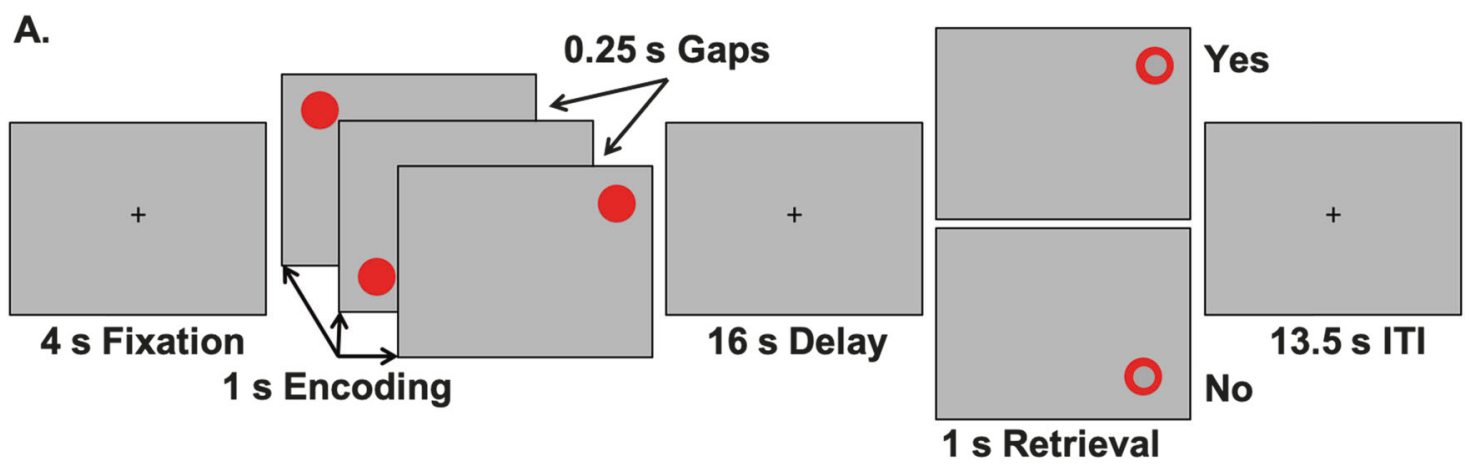

B.
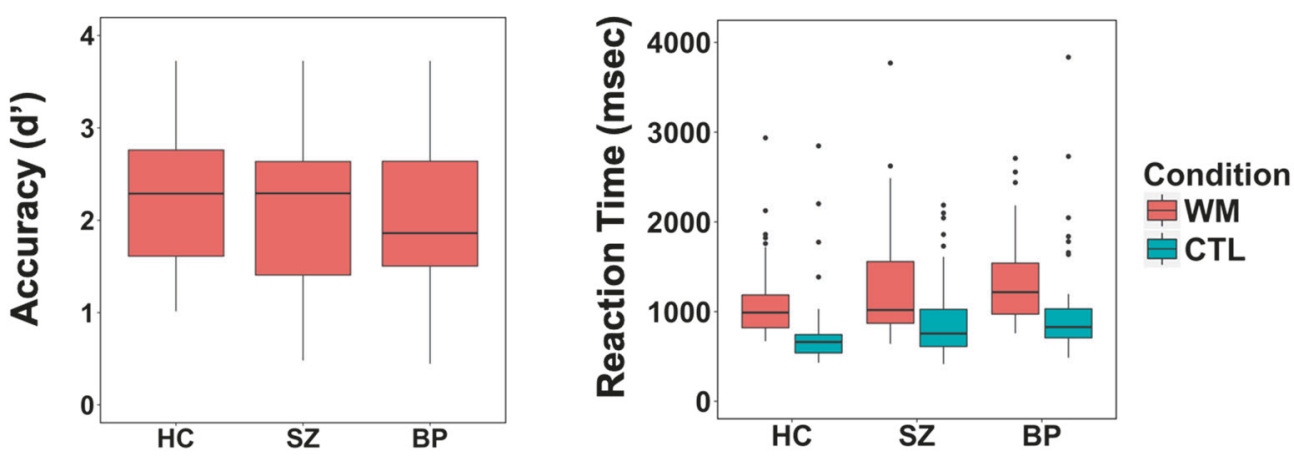

Fig. 1 a Schematic of the spatial delayed match-to-sample working memory task. $\mathbf{b}$ Accuracy (measured as d') and reaction time in working memory (WM) and control (CTL) condition for healthy controls (HC), schizophrenia (SZ) and Bipolar Disorder (BP) groups. b There were no differences between groups in accuracy. There was a significant difference in reaction time for both WM $(F(2,168)=3.55, p<.05)$ and $C T L$ conditions $(\mathrm{F}(2,168)=5.01, p<.01)$

$[18,19]$. For a detailed description of task parameters, see Supplemental Methods (Fig. 1a). Briefly, on WM trials, participants remembered the locations of 3 sequentially presented dots. After a $16 \mathrm{sec}$ delay, participants responded to a probe location with a button press indicating whether the probe appeared in one of the to be remembered locations or not. Sensorimotor control trials were identical to WM trials except that subjects were instructed to ignore the location of the dots and press both buttons simultaneously during the probe. Different colored dots were used to cue subjects to condition (red =WM; grey = Control).

Neuroimaging data acquisition and preprocessing

Imaging data were acquired on a $3 \mathrm{~T}$ Philips Intera Achieva scanner located at the Vanderbilt University Institute of Imaging Science during a single scanning session which included a T1weighted structural scan ( $1 \mathrm{~mm}^{3}$ isotropic voxel resolution) and up to 6 echo-planar imaging (EPI) functional scans $\left(3 \mathrm{~mm}^{3}\right.$ isotropic voxel resolution). Acquisition parameters and image processing are described in detail in the Supplemental Methods. Briefly, preprocessing steps included the following: 1) segmentation of T1-weighted images; 2) slice timing and motion correction of the EPI functional scans; 3) co-registration of EPI functional runs to native space T1-weighted images; 4) spatial normalization to Montreal Neurological Institute (MNI) space; and 5) spatial smoothing of the EPI functional data ( $6 \mathrm{~mm}$ FWHM).

\section{Functional neuroimaging data analysis}

First-level model definition. A General Linear Model (GLM) was created for each participant with regressors defined for three conditions: WM trials with correct responses, WM trials with incorrect responses, and control trials. Each trial was modeled with four stages: encoding (4-8s), early delay $(12-15 \mathrm{~s})$, late delay $(17-20 \mathrm{~s})$, and retrieval (24-25 s), convolved to canonical hemodynamic response functions. Onset times were relative to the initial fixation. The six motion parameters were included as covariates in the first-level model. We separately modeled earlyand late-delay periods based on neurophysiological studies, which showed that neurons with sustained activation across the delay period showed differential profiles. Some neurons showed a ramping down of activity across the delay, while others showed activity ramping up, thought to represent neural activity involved in stimulus encoding and preparation for action respectively [10]. For each individual, estimated parameters of the regressors (beta weights) were calculated for each voxel using the GLM and the resulting statistical maps from the contrast of WM correct trials only versus control trials for encoding, early delay, late delay and retrieval stages were used in the region-of-interest (ROI) and whole brain voxel-wise analyses described below.

Region-of-Interest Analyses. Consistent with prior studies using the same task, BOLD responses were extracted from $6 \mathrm{~mm}$ spherical ROls centered on the following frontal and parietal regions consistently implicated in spatial WM: (1) middle frontal gyrus (MFG); (2) anterior insula (Al); (3) frontal eye fields (FEF); (4) intraparietal sulcus (IPS); and (5) superior parietal lobule (SPL). Locations of the ROls, in MNI coordinates are presented in the Supplemental Methods. To identify specific coordinates for these ROls, we selected bilateral peak coordinates from the WM > Control trials contrast, during the early delay (across all subjects) that was closest to coordinates based on spatial WM literature in healthy individuals $[18,19,40]$. By selecting the coordinates from our task, we were choosing locations informed by the literature and showing maximal condition effect to investigate group differences. Average beta values for these ROls were extracted from the WM $>$ Control trials contrast for each subject at each stage. One-way ANOVAs were then performed for each stage and ROI separately with group entered as a between-subjects variable. Multiple comparisons were controlled using a Bonferroni adjusted $a$ based on 5 tests conducted (significance measured as $a \leq 0.01$ ). Post hoc differences were tested using least significant difference. 
Time courses for percent signal change were calculated to visualize activation across WM trials as described in the Supplemental Methods.

Whole Brain Analyses. The ROI analysis was complemented by a whole brain voxel-wise analysis. Briefly, individual subject WM > Control trials contrast maps for each stage were entered into separate one-way ANOVAs. Given our interest in group differences in task relevant brain regions, we masked the group analysis with the condition effect of WM > Control trials (threshold at voxel-wise $p<.05)$ for the corresponding stage using small volume cluster correction (SVC). Average betas from clusters surviving thresholding at $p<.05$ (FWE cluster-level corrected), voxel-wise threshold of $p<.001$, were extracted for post hoc analyses investigating differences between the three groups and for associations with task performance and neuropsychological functioning.

Association with task performance, neurocognition and clinical measures

Brain behavior correlation analyses focused on voxel-wise clusters from the whole brain analyses as these voxels showed the greatest difference between groups. Separate linear regression models that controlled for group membership were used to test associations between WM-related brain activation for each cluster and task performance, and each cluster and SCIP scores. Similarly, in analyses restricted to the two patient groups, separate linear regression models controlling for group were used to test associations between WM-related brain activations in each cluster and clinical measures (PANSS positive, negative and general scores) and medication (CPZ equivalents).

\section{RESULTS}

Behavioral Results

Task performance data are presented in Fig. $1 \mathrm{~b}$ and Supplementary Table 1. Accuracy (calculated as $d^{\prime}$ ) did not differ between groups $(F(2,167)=1.603, p=.204)$. For median reaction time (RT), main effects of condition $(F(2,167)=5.962, p=.003$; $W M>$ Control trials), and group $(F(2,167)=141.431, p<.001)$ were detected. The group $x$ condition interaction was not significant $(F(2,167)=0.135$, $p=.874$ ). Post hoc contrasts indicated that compared to healthy individuals, median RTs were longer in both schizophrenia $(p=.032)$ and bipolar disorder $(p=.006)$, with no difference between the two patient groups $(p=.358)$.

Neuroimaging Results: Region-of-Interest analyses

See Supplemental Table 2 for group mean beta values for each $\mathrm{ROI}$ and post hoc significance testing of group differences.

Encoding. A one-way ANOVA revealed significant group effects in the FEF $(F(2,168)=8.160, p<.001)$, SPL $(F(2,168)=12.088$, $p<.001)$ and IPS $(F(2,168)=8.758, p<.001)$ (Fig. 2). Post hoc comparisons indicated that activation in all three regions was higher in healthy individuals compared to both schizophrenia and bipolar disorder groups, but not significantly different between schizophrenia and bipolar disorder groups.

Maintenance. There were no significant group effects in any of the ROls during the early delay period. During the late delay period, the FEF and IPS showed significant differences between groups $(\mathrm{F}(2,168)=10.721, p<.001$ and $\mathrm{F}(2,168)=6.721, p=.002$ respectively) (Fig. 2). Post hoc analyses showed that healthy individuals had significantly greater activation in both regions compared to schizophrenia and bipolar disorder groups. The two patient groups did not significantly differ from each other.

Retrieval. There were no significant retrieval-related group effects in any of the pre-defined ROls.
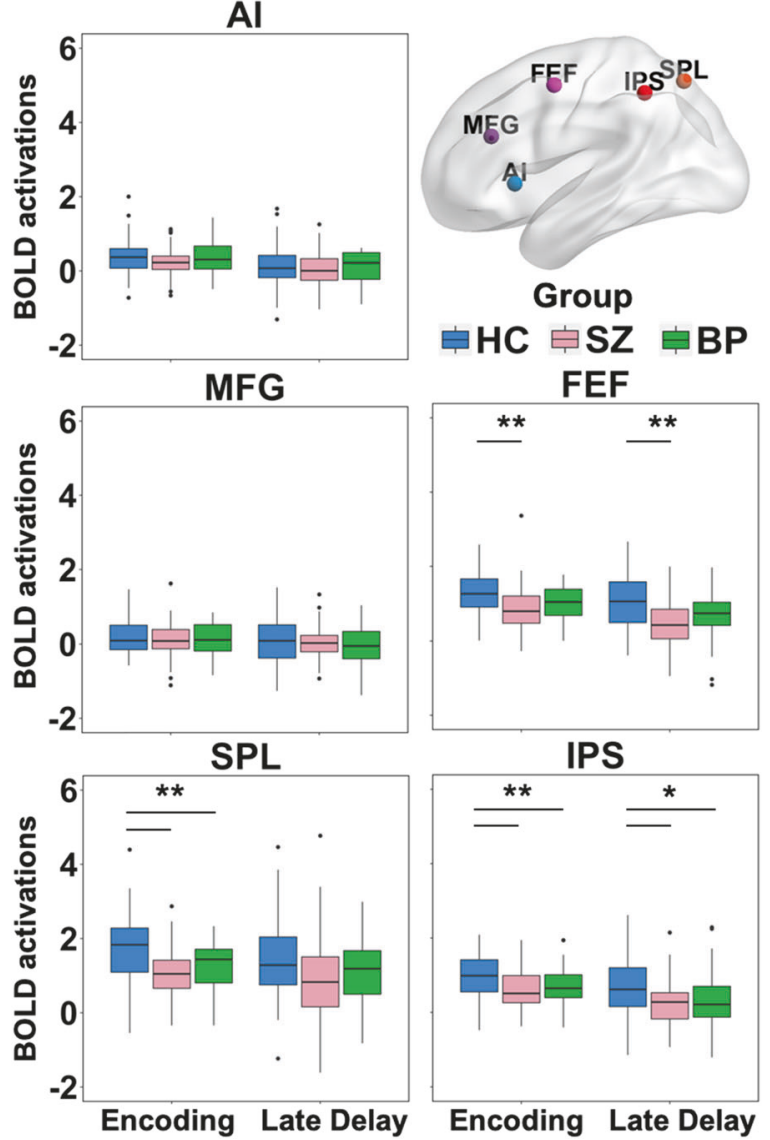

Fig. 2 BOLD activation (in arbitrary units) during WM encoding and late delay in frontal and parietal regions-of-interest (ROIs) commonly involved in spatial working memory. One-way ANOVA identified significant group effects in the intraparietal sulcus (IPS), superior parietal lobule (SPL) and frontal eye fields (FEF) during encoding, and IPS and FEF during the late delay $\left({ }^{*} p<.01,{ }^{* *} p<.001\right)$. Post hoc contrasts revealed that activation in these ROIs was reduced in schizophrenia (SZ) and psychotic bipolar disorder (BP) compared to healthy individuals $(\mathrm{HC})$. No significant group effects were seen in the anterior insula (AI) and middle frontal gyrus (MFG) during either encoding or late delay. The early delay and probe are not included in this figure as no significant group effects were found in any ROI during those stages

Neuroimaging results: whole brain analyses

Whole brain activations (i.e. WM > Control trials) within each group for the 4 WM stages are presented in Supplemental Table 3 and Supplemental Fig. 1-4 and 6-9. In brief, all three groups demonstrated qualitatively similar patterns of activation in a distributed set of frontoparietal and subcortical (thalamus, striatum) regions during $\mathrm{WM}$, with activations appearing more extensive during encoding and retrieval relative to maintenance, especially the late delay period. Group differences during each stage are summarized below.

Encoding. A one-way ANOVA revealed significant between group effects in clusters located in bilateral PPC and right ventral striatum (Fig. 3). Post hoc tests of extracted beta values indicated that bilateral PPC activation was greater in healthy individuals than both schizophrenia $(p<.001)$ and bipolar disorder groups $(p=.002)$, while the ventral striatum showed lower activation in the schizophrenia compared to both healthy $(p<.001)$ and bipolar disorder groups $(p<.001)$.

Maintenance. No regions showed significant differences between groups during the early delay period. During the late delay period, a 


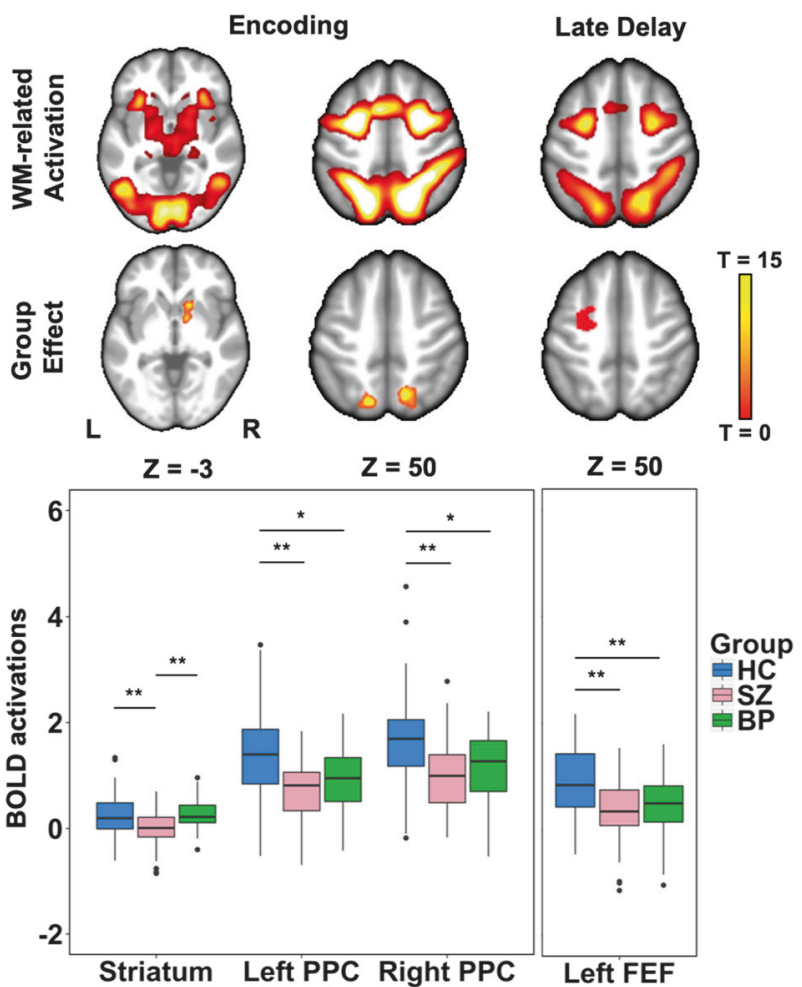

Fig. 3 One-way ANOVA of working memory correct $>$ control trial activation found group differences in the left and right posterior parietal cortex (PPC) and the right ventral striatum during encoding, and left frontal eye fields (FEF) during the late delay. Post hoc analyses performed on BOLD responses extracted from the clusters identified in the one-way ANOVA found both schizophrenia (SZ) and psychotic bipolar disorder (BP) groups exhibited reduced activation in bilateral PPC and left FEF, whereas only SZ demonstrated reduced activation in the right ventral striatum compared to both healthy individuals $(\mathrm{HC})$ and $\mathrm{BP}\left({ }^{*} p<.01,{ }^{* *} p<.001\right)$

one-way ANOVA showed significant group effects in the left FEF (Fig. 3). Post hoc tests of extracted beta values showed that the healthy group showed greater activation than both the schizophrenia $(p<.001)$ and bipolar disorder $(p<.001)$ groups.

Retrieval. No regions showed significant group effects during retrieval.

For post hoc analyses of whole brain differences between individual groups see Supplemental Results and Supplemental Table 4. See Fig. 4 for visualization of working memory trial time courses.

Correlations between brain function, WM task performance and neuropsychological functioning

Encoding-related activation in the right PPC and right ventral striatum clusters showed an association with task accuracy independent of group $\left(R_{\text {partial }}=.246, p<.001\right.$ and $R_{\text {partial }}=.234$, $p=.002$ respectively). Maintenance-related activation in the left FEF cluster also showed an association with task accuracy independent of group $\left(R_{\text {partial }}=.170, p=.027\right)$. While the brain-behavior associations did not always reach significance within each diagnostic group, the direction of the associations were consistent and did not significantly differ across groups. With respect to general neuropsychological functioning, assessed with the SCIP, encoding-related activation in the right PPC showed an association with SCIP composite $z$-score, independent of group $\left(R_{\text {partial }}=.155, p=.045\right)$. Exploratory analyses of correlations between activation in predefined ROls indicate that encoding-, early delay- and late delay-
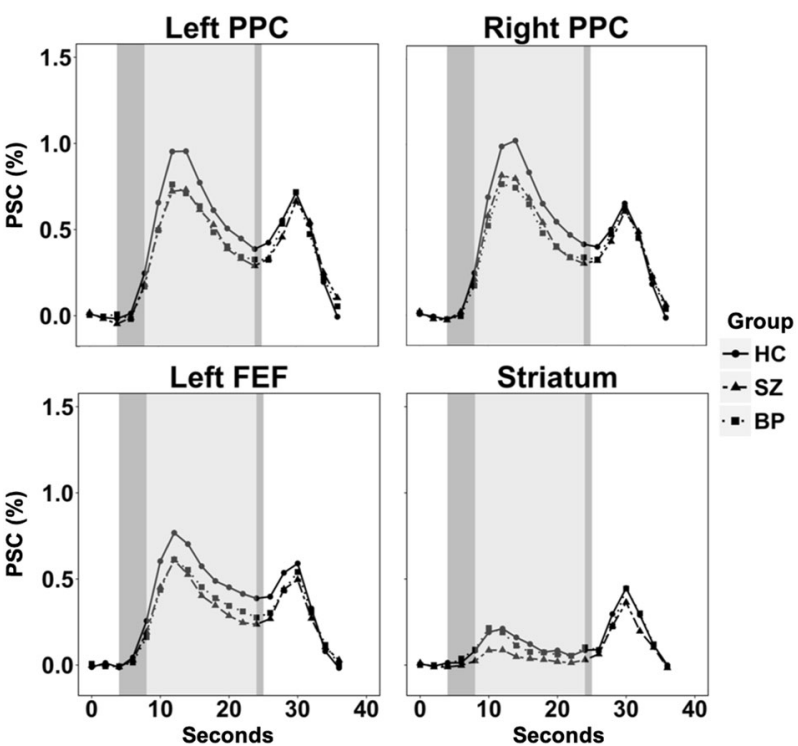

Fig. 4 Visualization of the time course of percent signal change (PSC) across working memory trials for the regions showing significant group effects in the whole brain analysis

related activation in the IPS, SPL and FEF were all positively associated with task performance (Supplemental Table 5).

Correlation between brain function, clinical symptomatology and medication

PANSS general score correlated negatively with encoding-related activation in the left PPC $\left(R_{\text {partial }}=-0.208, p=0.028\right)$, and maintenance-related activation in the left FEF $\left(R_{\text {partial }}=-0.195\right.$, $p=0.039$ ), independent of diagnosis. There were no other significant associations between brain activations and PANSS positive, negative, general scores or medication.

\section{DISCUSSION}

WM is impaired in psychotic disorders, related to genetic risk for psychosis, and associated with functional outcome [3-8]. Relatively little is known about the neural correlates of specific WM stages in psychosis as most prior neuroimaging investigations used tasks that inherently mix encoding, maintenance, and retrieval and/or place significant demands on executive functions. To avoid these limitations, we used a DMTS spatial WM task well-suited to investigating separable WM stages with minimal executive function demands. Examination of a-priori defined ROls identified reduced encoding- and maintenance-related activity in the PPC (IPS and SPL) and FEF in both schizophrenia and psychotic bipolar disorder. Complimentary whole brain analyses confirmed reduced activity in the PPC and FEF across psychotic disorders and revealed additional reduction in right ventral striatal activation during WM encoding that was specific to schizophrenia. Finally, activation in several regions that demonstrated reduced WM-related activation in psychosis, particularly the PPC, correlated with task performance and overall neuropsychological functioning.

At first glance, our results appear inconsistent with both the broader WM neuroimaging literature in psychosis and prior investigations using similar tasks, which have implicated PFC dysfunction in WM impairment. For example, a prior study using the same spatial WM paradigm reported reduced activation during WM maintenance in several PFC regions, including the dorsolateral PFC [19]. Similarly, Anticevic and colleagues [24] identified reduced activation in anterior insula, medial PFC, and dorsolateral PFC during both encoding and maintenance. Several factors might explain the discrepancy. First, we examined a slightly different set 
of regions from previous studies, including two PPC regions (IPS and $\mathrm{SPL}$ ) that were not examined in some prior studies that focused exclusively on PFC ROls, therefore allowing us to find differences in parietal regions (e.g. [19]). Second, we isolated brain regions involved in WM by contrasting WM trials to a sensorimotor control condition rather than a low-level fixation baseline, which represents memory related activation independent of sensorimotor responses $[19,24]$. Finally, the sample sizes included in our study are much larger than most prior studies. For instance, the earlier study by Driesen and colleagues [19] included 12 healthy individuals and 14 individuals with psychosis, compared to 58 and 72 in the current investigation.

While our findings are somewhat at odds with PFC-centric models of WM impairment in psychosis, they dovetail nicely with evidence that the PPC plays a crucial role in encoding and maintaining visual information in WM [41, 42]. Specifically, findings in healthy individuals suggest that the PFC is primarily involved in executive functions supporting higher order WM processes, such as rule maintenance and response inhibition, while the PPC is proposed to underlie the attention and storage components of WM [31]. A recent investigation of WM storage indicates that there may be distinct core deficits in schizophrenia, separately focused in PFC regions underlying executive function processes, and in the PPC supporting WM storage [32]. Our results are consistent with this view, further indicating that this PPC impairment occurs early during the memory process, during initial encoding and maintenance. This is consistent with behavioral work suggesting that WM deficits are prominent during encoding and early maintenance stages of DMTS tasks [43] and that attention capture by task irrelevant stimuli at encoding contribute to WM impairment in psychosis [44]. Interestingly, PPC and FEF activations were associated with task performance; individuals with greater activation in these regions also showed greater task accuracy. This effect was independent of any mean group differences, suggesting that decreased activation in these regions in patients may drive differences in WM ability. Further, encodingrelated activation in the PPC was associated with general neuropsychological functioning. This finding is consistent with a previous report which showed that both healthy individuals and those with schizophrenia demonstrated an association between PPC function and MATRICS Consensus Cognitive Battery scores, another measure of general cognitive function [32] and is consistent with the view that PPC deficits have broader consequences on cognition in psychosis. While consistent with Hahn and colleagues [32], our brain-behavior correlations were less robust, suggesting that parametric designs may better probe activation associated with general cognition.

The finding that psychotic bipolar disorder and schizophrenia demonstrate very similar deficits in WM network regions, with both groups demonstrating lower encoding- and maintenance-related activation in PPC and FEF, has important implications for understanding the etiology and treatment of WM impairment in psychotic disorders. In terms of etiology, these results suggest that across psychotic disorders, there are impairments in the neurobiological substrates supporting WM storage, indicating that executive function deficits are not the only commonality between different psychotic disorders. From a treatment perspective, our findings suggest that interventions targeting encoding and maintenance may be most effective, potentially paired with modern techniques that are able to enhance neural activity such as repeated transcranial magnetic stimulation (rTMS) [45]. Moreover, evidence that PPC activation during WM correlates with broad neuropsychological functions suggests that interventions improving PPC dependent WM processes may generalize to other cognitive abilities.

The only brain region that differed between schizophrenia and psychotic bipolar disorder was the right ventral striatum. This region showed deficits in schizophrenia, but not psychotic bipolar disorder and this effect was only present at encoding. The ventral striatum is involved in reward and motivation behaviors [46] and is generally not considered part of the core WM network [40, 47]. It is possible that our finding of decreased striatal activation was associated with reduced motivation to encode in our schizophrenia group, though no group differences in mean performance were observed. However, we did find a positive correlation between task performance and activation, independent of group. The striatum has been conceptualized as important in the pathogenesis of cognitive symptoms in schizophrenia, particularly in association with the dopamine hypothesis [48], and studies have shown decreased activation in schizophrenia compared with healthy individuals [49]. In bipolar disorder, a meta-analysis found a similar region in the left ventral striatum that showed greater activation compared with healthy individuals across a host of functional neuroimaging paradigms that included cognitive, affective and reward based tasks [50]. Ventral striatal differences may also be due to increased baseline activation or an effect of antipsychotic medication, although the difference between patient groups persisted after adjusting for medication dosage.

Our study has several limitations that must be considered when interpreting the results. Notably, the paradigm used in this study was chosen to minimize executive control requirements to better investigate specific stages of WM relatively independently of executive functions. Consequently, the fact that we did not find significant impairment in PFC activations does not imply that PFC functions are intact. To the contrary, there is abundant evidence that PFC recruitment during executive cognitive tasks is abnormal in psychosis $[16,17]$. Another limitation was that we did not include the ventrolateral PFC in our analysis, a region that is commonly considered part of the WM network, and has been investigated in previous DMTS designs $[19,24]$. We chose to exclude the ventrolateral PFC from our ROI analyses as it did not show a significant condition effect (i.e. WM > Control trials during the early delay) across subjects. In addition, our task did not allow us to separate true encoding from early maintenance due to our choice of sequential stimulus presentation. A task using simultaneous stimulus presentation, such as Hahn and colleagues [32] used, with separable working memory stages would be better suited to address differences between encoding and early maintenance. Also, we only examined correct WM trials, therefore, to accurately estimate first-level GLM parameters, we excluded our lower performing participants. This may affect how representative our samples were. Finally, most of our individuals with schizophrenia and psychotic bipolar disorder were medicated (Supplementary Table 6), and less stringent substance use disorder exclusion criteria were used for individuals with psychotic disorder, therefore we cannot rule out the effect of medication or substance use on our results.

In conclusion, our findings highlight the importance of brain areas outside the dorsolateral PFC, primarily FEF and PPC, in WM impairment in both schizophrenia and psychotic bipolar disorder. Interventions targeting these regions and specific WM processes, encoding and maintenance in particular, may be fruitful avenues to pursue for treating cognitive impairment in psychotic disorders.

\section{FUNDING AND DISCLOSURE}

This work was supported by NIMH grant R01 MH102266 (awarded to NDW), the Charlotte and Donald Test Fund, the Jack Martin, MD, Research Professorship in Psychopharmacology (awarded to JUB), the Vanderbilt Psychiatric Genotype/Phenotype Project, and the Vanderbilt Institute for Clinical and Translational Research (through grant 1-UL-1-TR000445 from the National Center for Research Resources/NIH). JUB was partially supported by a Merit award (No. CX001226). This work was conducted in part using the resources of the Advanced Computing Center for Research and Education at Vanderbilt University, Nashville, TN. No commercial support was received for the preparation of this manuscript. The authors declare no competing interests. 


\section{ADDITIONAL INFORMATION}

Supplementary Information accompanies this paper at (https://doi.org/10.1038/ s41386-019-0434-4).

Publisher's note: Springer Nature remains neutral with regard to jurisdictional claims in published maps and institutional affiliations.

\section{REFERENCES}

1. Simonsen $C$, Sundet $K$, Vaskinn A, Birkenaes $A B$, Engh JA, Faerden $A$, et al. Neurocognitive dysfunction in bipolar and schizophrenia spectrum disorders depends on history of psychosis rather than diagnostic group. Schizophr Bull. 2011;37:73-83.

2. Menkes MW, Armstrong K, Blackford JU, Heckers S, Woodward ND. Neuropsychological functioning in early and chronic stages of schizophrenia and psychotic bipolar disorder. Schizophr Res. 2018. https://doi.org/10.1016/j. schres.2018.10.009.

3. Gold Jo, Barch DM, Feuerstahler LM, Carter CS, MacDonald AW III, Ragland JD, et al. Working memory impairment across psychotic disorders. Schizophr Bull. 2018. https://doi.org/10.1093/schbul/sby134.

4. Knowles EEM, Carless MA, de Almeida MAA, Curran JE, McKay DR, Sprooten E, et al. Genome-wide significant localization for working and spatial memory: Identifying genes for psychosis using models of cognition. Am J Med Genet B Neuropsychiatr Genet. 2014;165B:84-95.

5. Glahn DC, Therman S, Manninen $M$, Huttunen $M$, Kaprio J, Lönnqvist J, et al. Spatial working memory as an endophenotype for schizophrenia. Biol Psychiatry. 2003;53:624-6.

6. Tuulio-Henriksson A, Haukka J, Partonen T, Varilo T, Paunio T, Ekelund J, et al. Heritability and number of quantitative trait loci of neurocognitive functions in families with schizophrenia. Am J Med Genet. 2002;114:483-90.

7. Cannon TD, Huttunen MO, Lonnqvist J, Tuulio-Henriksson A, Pirkola T, Glahn D, et al. The inheritance of neuropsychological dysfunction in twins discordant for schizophrenia. Am J Hum Genet. 2000;67:369-82.

8. Park S, Holzman PS, Goldman-Rakic PS. Spatial working memory deficits in the relatives of schizophrenic patients. Arch Gen Psychiatry. 1995;52:821-8.

9. Watanabe M. Cognitive and motivational operations in primate prefrontal neurons. Rev Neurosci. 1998;9:225-41.

10. Funahashi S, Chafee MV, Goldman-Rakic PS. Prefrontal neuronal activity in rhesus monkeys performing a delayed anti-saccade task. Nature. 1993;365:753-6.

11. Funahashi S, Kubota K. Working memory and prefrontal cortex. Neurosci Res. 1994:21:1-11.

12. Goldman-Rakic PS. Working memory dysfunction in schizophrenia. J Neuropsychiatry Clin Neurosci. 1994;6:348-57.

13. Goldman-Rakic PS, Selemon LD. Functional and anatomical aspects of prefrontal pathology in schizophrenia. Schizophr Bull. 1997:23:437-58.

14. Lewis DA, Gonzalez-Burgos G. Intrinsic excitatory connections in the prefrontal cortex and the pathophysiology of schizophrenia. Brain Res Bull. 2000;52:309-17.

15. Krystal JH, Anticevic A, Yang GJ, Dragoi G, Driesen NR, Wang X-J, et al. Impaired tuning of neural ensembles and the pathophysiology of schizophrenia: a translational and computational neuroscience perspective. Biol Psychiatry. 2017;81:874-85.

16. Minzenberg MJ, Laird AR, Thelen S, Carter CS, Glahn DC. Meta-analysis of 41 functional neuroimaging studies of executive function in schizophrenia. Arch Gen Psychiatry. 2009;66:811-22.

17. Glahn DC, Ragland JD, Abramoff A, Barrett J, Laird AR, Bearden CE, et al. Beyond hypofrontality: a quantitative meta-analysis of functional neuroimaging studies of working memory in schizophrenia. Hum Brain Mapp. 2005;25:60-9.

18. Leung H-C, Gore JC, Goldman-Rakic PS. Sustained mnemonic response in the human middle frontal gyrus during on-line storage of spatial memoranda. J Cogn Neurosci. 2002;14:659-71.

19. Driesen NR, Leung H-C, Calhoun VD, Constable RT, Gueorguieva R, Hoffman R, et al. Impairment of working memory maintenance and response in schizophrenia: functional magnetic resonance imaging evidence. Biol Psychiatry. 2008;64:1026-34.

20. Manoach DS, Gollub RL, Benson ES, Searl MM, Goff DC, Halpern E, et al. Schizophrenic subjects show aberrant $\mathrm{fMRI}$ activation of dorsolateral prefrontal cortex and basal ganglia during working memory performance. Biol Psychiatry. 2000;48:99-109.

21. Tan $\mathrm{H}-\mathrm{Y}$, Choo W-C, Fones CSL, Chee MWL. fMRI study of maintenance and manipulation processes within working memory in first-episode schizophrenia. Am J Psychiatry. 2005;162:1849-58.

22. Lee J, Folley BS, Gore J, Park S. Origins of spatial working memory deficits in schizophrenia: an event-related FMRI and near-infrared spectroscopy study. PLoS ONE. 2008;3:e1760.
23. Luck D, Danion J-M, Marrer C, Pham B-T, Gounot D, Foucher J. Abnormal medial temporal activity for bound information during working memory maintenance in patients with schizophrenia. Hippocampus. 2010;20:936-48.

24. Anticevic A, Repovs G, Barch DM. Working memory encoding and maintenance deficits in schizophrenia: neural evidence for activation and deactivation abnormalities. Schizophr Bull. 2011:39:168-78.

25. Avsar KB, Stoeckel LE, Bolding MS, White DM, Tagamets MA, Holcomb HH, et al. Aberrant visual circuitry associated with normal spatial match-to-sample accuracy in schizophrenia. Psychiatry Res. 2011;193:138-43.

26. Schlösser RGM, Koch K, Wagner G, Nenadic I, Roebel M, Schachtzabel C, et al. Inefficient executive cognitive control in schizophrenia is preceded by altered functional activation during information encoding: an fMRI study. Neuropsychologia. 2008;46:336-47.

27. Wible CG, Lee K, Molina I, Hashimoto R, Preus AP, Roach BJ, et al. fMRI activity correlated with auditory hallucinations during performance of a working memory task: data from the FBIRN consortium study. Schizophr Bull. 2009;35:47-57.

28. Kim J, Matthews NL, Park S. An event-related FMRI study of phonological verbal working memory in schizophrenia. PLoS ONE. 2010;5:e12068.

29. van Veelen NMJ, Vink M, Ramsey NF, van Buuren M, Hoogendam JM, Kahn RS. Prefrontal lobe dysfunction predicts treatment response in medication-naive first-episode schizophrenia. Schizophr Res. 2011;129:156-62.

30. Bittner RA, Linden DEJ, Roebroeck A, Härtling F, Rotarska-Jagiela A, Maurer K, et al. The when and where of working memory dysfunction in early-onset schizophrenia-a functional magnetic resonance imaging study. Cereb Cortex. 2015;25:2494-506.

31. D'Esposito M, Postle BR. The cognitive neuroscience of working memory. Annu Rev Psychol. 2015;66:115-42.

32. Hahn B, Robinson BM, Leonard CJ, Luck SJ, Gold JM. Posterior parietal cortex dysfunction is central to working memory storage and broad cognitive deficits in schizophrenia. J Neurosci. 2018;38:8378-87.

33. Brandt CL, Eichele T, Melle I, Sundet K, Server A, Agartz I, et al. Working memory networks and activation patterns in schizophrenia and bipolar disorder: comparison with healthy controls. Br J Psychiatry. 2014;204:290-8.

34. Hamilton LS, Altshuler LL, Townsend J, Bookheimer SY, Phillips OR, Fischer J, et al. Alterations in functional activation in euthymic bipolar disorder and schizophrenia during a working memory task. Hum Brain Mapp. 2009;30:3958-69.

35. First MB, Gibbon M, Spitzer RL, Williams JBW. User's guide for the structured clinical interview for DSM-IV axis I Disorders-Research version. New York, NY: Biometrics Research Department, New York State Psychiatric Institute; 1996.

36. Kay SR, Fiszbein A, Opler LA. The positive and negative syndrome scale (PANSS) for schizophrenia. Schizophr Bull. 1987;13:261-76.

37. Young RC, Biggs JT, Ziegler VE, Meyer DA. A rating scale for mania: reliability, validity and sensitivity. Br J Psychiatry. 1978;133:429-35.

38. Wechsler D. Wechsler test of adult reading: WTAR. Psychological Corporation, San Antonio, TX; 2001.

39. Purdon SE. The Screen for Cognitive Impairment in Psychiatry (SCIP): Instructions and three alternate forms. Alberta: PNL Inc, Edmonton; 2005. p. 2005.

40. Rottschy C, Langner R, Dogan I, Reetz K, Laird AR, Schulz JB, et al. Modelling neural correlates of working memory: a coordinate-based meta-analysis. Neuroimage. 2012;60:830-46.

41. Todd JJ, Marois R. Capacity limit of visual short-term memory in human posterior parietal cortex. Nature. 2004;428:751-4

42. Tseng $\mathrm{P}$, Hsu T-Y, Muggleton NG, Tzeng OJL, Hung DL, Juan C-H. Posterior parietal cortex mediates encoding and maintenance processes in change blindness. Neuropsychologia. 2010:48:1063-70.

43. Lee J, Park S. Working memory impairments in schizophrenia: a meta-analysis. J Abnorm Psychol. 2005;114:599-611.

44. Mayer JS, Fukuda K, Vogel EK, Park S. Impaired contingent attentional capture predicts reduced working memory capacity in schizophrenia. PLoS ONE. 2012;7: e48586.

45. Reinhart RMG, Nguyen JA. Working memory revived in older adults by synchronizing rhythmic brain circuits. Nat Neurosci. 2019;22:820-827.

46. Schultz W, Dayan P, Montague PR. A neural substrate of prediction and reward. Science. 1997;275:1593-9.

47. Owen AM, McMillan KM, Laird AR, Bullmore E. N-back working memory paradigm: a meta-analysis of normative functional neuroimaging studies. Hum Brain Mapp. 2005;25:46-59.

48. Simpson EH, Kellendonk C, Kandel E. A possible role for the striatum in the pathogenesis of the cognitive symptoms of schizophrenia. Neuron. 2010;65:585-96.

49. Walter $\mathrm{H}$, Vasic N, Höse A, Spitzer M, Wolf RC. Working memory dysfunction in schizophrenia compared to healthy controls and patients with depression: evidence from event-related fMRI. Neuroimage. 2007;35:1551-61.

50. Chen $\mathrm{C}-\mathrm{H}$, Suckling J, Lennox BR, Ooi $\mathrm{C}$, Bullmore ET. A quantitative meta-analysis of fMRI studies in bipolar disorder. Bipolar Disord. 2011;13:1-15. 